\title{
Distinct metabolic preference of atypical KRAS mutant
}

\author{
Heesun Cheong \\ Division of Cancer Biology, National Cancer Center, Goyang, Republic of Korea \\ Correspondence to: Heesun Cheong. Division of Cancer Biology, National Cancer Center, 323 Ilsan-ro, Ilsandong-gu, Goyang, Gyeonggi-do 10408, \\ Republic of Korea. Email: heesunch@ncc.re.kr. \\ Comment on: Hobbs GA, Baker NM, Miermont AM, et al. Atypical KRAS G12R Mutant Is Impaired in PI3K Signaling and Macropinocytosis in \\ Pancreatic Cancer. Cancer Discov 2020;10:104-23.
}

Submitted Apr 10, 2020. Accepted for publication Apr 28, 2020.

doi: 10.21037/atm-20-3252

View this article at: http://dx.doi.org/10.21037/atm-20-3252

Pancreatic ductal adenocarcinoma (PDAC) is an extremely lethal form of cancer that typically results from small GTPase KRAS point mutations. These constitutively active mutants are prone to the activation of multiple downstream effectors, which transduces growth-related signals to the cells and promotes cellular neoplastic phenotypes. Various types of KRAS mutations have been reported in almost $30 \%$ of all cancers including $95 \%$ of PDAC, $35 \%$ of lung adenocarcinoma, and $40 \%$ of colon cancers. Most point mutations of KRAS occur in three major amino acid residues G12, G13, or Q61, resulting in GTP bound active form of KRAS (1). The position of KRAS mutations varies among cancers in different tissues: PDAC and non-small cell lung carcinoma (NSCLC) showed KRAS mutations at G12 most frequently, whereas colon and rectal carcinoma (CRC) showed the additional G13 mutation ( $20 \%)$, as well as predominant point mutation at G12. Moreover, the types of substitution at G12 are also dependent on tissue types; for example, the predominant substitutions of G12 in PDAC and CRC are G12D and G12V. In contrast, the major substitution in NSCLC is G12C, which is rare in PDAC. Another type of substitution, G12R is rarely found in colorectal cancer and lung cancer $(\sim 1 \%)$, but more frequently shown in PDAC ( 12\%) (1). Understanding physiology and molecular regulation of distinct KRAS mutations in each context would provide fundamentals to develop personalized anti-cancer therapies for targeting mutant specific KRAS.

Recently, Hobbs et al. (2) have reported that distinct point mutations of KRAS confer context specific effects on cell growth and survival, particularly in PDAC development. First, the authors have examined the molecular significance of KRAS G12R mutant compared to that of common KRAS G12D and G12V in aspect of multiple growth-related downstream signaling pathways. Reverse-phase protein array (RPPA) analyses and immunoblots for phosphorylation of KRAS downstream effectors were performed in cells expressing a series of KRAS G12 mutants, frequently shown in PDAC. As a result, PI3K-AKT signals was significantly downregulated in the KRAS G12R mutants-expressing cells, compared to that of KRAS G12D or G12V mutants. Interestingly, the authors found that phosphor AKT levels in KRAS G12R mutant-expressing epithelial cells were significantly reduced, but still intact in KRAS G12D mutant.

They also found that KRAS G12R mutant failed to interact with an important subunit of PI3K p110 $\alpha$ (PIK3CA), a well-known KRAS downstream target, because of its unordered protein structure in switch II region to complete interaction with PI3K p110 $\alpha$. This result was confirmed by their in-depth structural analysis of G12R mutant protein. As the importance of KRAS and PI3K p110 $\alpha$ interaction in tumor progression has been reported previously in mice (3), KRAS G12R mutants can be expected to show substantial reduction of cell growth, explaining why this atypical KRAS mutant is shown to be relatively rare in lung and colon cancers.

However, the authors raised questions about how KRAS G12R mutant can still lead cell proliferation and sustain high prevalence in PDAC development, despite its 
low PI3K $\alpha$ activity. One hypothesis is that KRAS G12R mutant may rewire specific downstream signaling pathways associated with KRAS activity thus this mutant might utilize alternative pathways to support PDAC development.

Given that nutrients-supporting for cancer cell growth was supplied by oncogenic KRAS-mediated macropinocytosis, the atypical point mutation of KRAS, G12R mutant was examined to determine its importance for macropinocytosis. PDAC cell lines harboring KRAS G12D and G12V showed decreasing macropinocytosis under transient KRAS knockdown. In contrast, multiple PDAC cell lines containing KRAS G12R still promote macropinocytosis in knockdown of KRAS despite the defective PI3K $\alpha$ activity.

Macropinocytosis is a unique clathrin-independent endocytic process that internalizes macromolecule-rich extracellular fluid without the need for specific vesiclecoat proteins. Multiple growth factor signals are known to induce macropinocytosis, which facilitates the protrusion of plasma membrane ruffles, allowing engulfed extracellular fluid to be internalized into the cell. This process is most well-defined in dendritic cells and macrophages. Recently, macropinocytosis has been revealed to contribute to cell growth and survival in cancer cells through the maintenance of nutrient balance $(4,5)$. The expression of oncogenic Ras including KRAS and HRAS has been reported to induce macropinocytosis, which plays a major role in the support of the cellular demand for metabolites, such as amino acids, during the metabolic stress resulting from the rapid growth of cancers (6).

When three different types of KRAS G12 mutant were overexpressed in normal epithelial cells respectively, KRAS G12D or G12V mutants- tended to promote macropinocytosis whereas KRAS G12R mutant showed reduced levels of macropinocytosis. However, multiple PDAC cells harboring KRAS G12R mutant still showed sufficient macropinocytosis even in the knockdown of KRAS, suggesting that other mechanisms might be regulating factors for inducing macropinocytosis in addition to KRAS. Of interest, co-expression of a constitutively activated PI3K p110 $\alpha$ variant (p110 $\alpha$ CAAX) (7) with various G12 mutants, significantly rescued macropinocytosis, especially in KRAS G12R mutantexpressing cells, suggesting that PI3K-AKT signaling plays a positive role in regulate macropinocytosis as well as cell growth. Conclusively, the lack of interaction with PI3K, p110 $\alpha$ can lead to the impairment of KRAS G12R to induce macropinocytosis.
Although the authors have discussed that tumor cells harboring same mutant can reveal their various phenotypes in a context with genetic and epigenetic complexity, each point mutation in KRAS, a pivotal oncogenic driver, can determine cell physiology by regulating metabolic sensitivity of cancer cells in multiple layer networks. Recently, our findings (8) with other recent reports (9), suggested that mTOR plays distinctive roles in regulating macropinocytosis in environmental- or context-dependent manner. Under normal nutrient conditions, PI3K-mTOR activity has a predominant role in transmitting growthrelated signaling to support cell growth and survival. PI3K works as a positive effector for macropinocytosis which is dispensable for cell growth under nutrient-rich conditions. However, when mTOR activity is suppressed under nutrient-poor, metabolic stress conditions, macropinocytosis becomes an essential route to support cell growth and survival. Accordingly, it is presumed that KRAS G12R mutant PDAC cell lines decreases mTOR activities, thereby preferentially utilizing macropinocytosis to generate energy supply and sustain tumor progression.

Moreover, the suppression of PI3 $\mathrm{K} \alpha$ activity in KRAS G12R mutant PDAC cells might induce catabolic signaling pathways such as autophagy to maintain cellular metabolic homeostasis. Indeed, on examination of basal autophagy activity among in KRAS G12 mutant PDAC cells, KRAS G12V and KRAS G12D mutants showed substantially reduced autophagy activity rather than that of KRAS G12R mutant.

It has been reported that PI3K-AKT and ERK/MAPK, well-known downstream effectors of active KRAS, have a compensatory regulation mechanism between these two signaling pathways (10). Thus, it can be predicted that defective PI3K $\alpha$ activity caused by KRAS G12R mutation confers sensitivity to inhibition of ERK/MAPK. Based on this rationale, initially the authors have performed large scale cytotoxicity analyses in several KRAS G12R and G12D PDAC cell lines with single inhibitors. As expected KRAS G12R mutant PDAC lines tend to be more sensitive to either MEK inhibitor or autophagy inhibitor-chloroquine than KRAS G12D mutant. Moreover, PDAC organoids and PDAC patient-derived xenograft (PDX) tumors with KRAS G12R mutant are also more susceptible to treatment with MEK1/2-selective inhibitor, Selumetinib.

Overall data suggest that KRAS G12R mutant PDAC more likely utilizes a degradative pathway, autophagy to support cancer growth, which was induced by defective PI3K-AKT growth signaling in KRAS G12R mutation. 
Therefore, combinatorial treatment of ERK inhibitor and chloroquine can provides synergistic anti-cancer effect, particularly in KRAS G12R mutant PDAC cell lines, but just additive effect in KRAS G12D mutant. In addition to lysosomotropic autophagy inhibitors, specific pharmacological inhibitors of ULK1s, an autophagyrelated protein, also exhibited synergistic anti-tumorigenic effect in combination with ERK inhibitors. Although the similar antitumor strategy using combinatorial treatment of ERK and autophagy inhibitors in pancreatic cancer has been reported (11), the authors more likely focused on the distinct molecular roles of allele-specific KRAS mutants in this study.

This work delineates a molecular mechanism to understand how specific types of KRAS mutation redirect downstream signaling pathways to access metabolically favorable cellular processes which ultimately support rapid tumor progression; for example, PDAC harboring KRAS G12R mutant seems more addicted to lysosomal degradation of intra- or extracellular macromolecules following autophagy or macropinocytosis under the suppressive conditions of $\mathrm{PI} 3 \mathrm{~K} \alpha-\mathrm{mTOR}$ signaling axis. Accordingly, for PDAC harboring atypical KRAS G12R mutation, concomitant targeting macropinocytosis and ERK/MEK pathway can be considered as a promising anti-cancer strategy, which should be investigated further. Moreover, the results of this study might also provide a molecular basis for setting up guidance to patient stratification for personalized cancer treatment.

Furthermore, it should be worthwhile to generate atypical mutation KRAS G12R-driven PDAC mouse model which is used for further investigation in vivo to examine whether this mouse model derived from pancreasspecific mutations KRAS G12R can develop PDAC, with or without a well-known additional mutant p53. This spontaneous PDAC mouse model would be important in vivo system to examine the physiological role and regulation mechanism of macropinocytosis in PDAC derived from KRAS G12R mutation. In addition, this system would be useful to estimate in vivo therapeutic potency of those having multiple anti-cancer approaches, which should be determined prior to personalized clinical trials.

\section{Acknowledgments}

Funding: This study was supported by National Research Foundation of Korea grant funded by the Ministry of Education of Korea (NRF-2020R1A2B5B01002011).

\section{Footnote}

Provenance and Peer Review: This article was commissioned and reviewed by the Section Editor Le Li, MD, PhD (Department of Pancreatic and Biliary Surgery, The First Affiliated Hospital of Harbin Medical University, Harbin Medical University, Harbin, China).

Conflicts of Interest: The author has completed the ICMJE uniform disclosure form (available at http://dx.doi. org/10.21037/atm-20-3252). The author has no conflicts of interest to declare.

Ethical Statement: The author is accountable for all aspects of the work in ensuring that questions related to the accuracy or integrity of any part of the work are appropriately investigated and resolved.

Open Access Statement: This is an Open Access article distributed in accordance with the Creative Commons Attribution-NonCommercial-NoDerivs 4.0 International License (CC BY-NC-ND 4.0), which permits the noncommercial replication and distribution of the article with the strict proviso that no changes or edits are made and the original work is properly cited (including links to both the formal publication through the relevant DOI and the license). See: https://creativecommons.org/licenses/by-nc-nd/4.0/.

\section{References}

1. Cox AD, Fesik SW, Kimmelman AC, et al. Drugging the undruggable RAS: Mission possible? Nat Rev Drug Discov 2014;13:828-51.

2. Hobbs GA, Baker NM, Miermont AM, et al. Atypical KRAS G12R Mutant Is Impaired in PI3K Signaling and Macropinocytosis in Pancreatic Cancer. Cancer Discov 2020;10:104-23.

3. Gupta S, Ramjaun AR, Haiko P, et al. Binding of ras to phosphoinositide 3-kinase p110alpha is required for rasdriven tumorigenesis in mice. Cell 2007;129:957-68.

4. Bar-Sagi D, Feramisco JR. Induction of membrane ruffling and fluid-phase pinocytosis in quiescent fibroblasts by ras proteins. Science 1986;233:1061-8.

5. Mercer J, Helenius A. Virus entry by macropinocytosis. Nat Cell Biol 2009;11:510-20.

6. Commisso C, Davidson SM, Soydaner-Azeloglu RG, et al. Macropinocytosis of protein is an amino acid supply route in Ras-transformed cells. Nature 2013;497:633-7. 


\section{Page 4 of 4}

7. McFall A, Ulkü A, Lambert QT, et al. Oncogenic Ras blocks anoikis by activation of a novel effector pathway independent of phosphatidylinositol 3-kinase. Mol Cell Biol 2001;21:5488-99.

8. Sung S, Choi J, Cheong H. Catabolic pathways regulated by mTORC1 are pivotal for survival and growth of cancer cells expressing mutant Ras. Oncotarget 2015;6:40405-17.

9. Palm W, Park Y, Wright K, et al. The Utilization of

Cite this article as: Cheong H. Distinct metabolic preference of atypical KRAS mutant. Ann Transl Med 2020;8(20):1326. doi: 10.21037/atm-20-3252

\section{Cheong. Macropinocytosis regulation of atypical KRAS mutant}

Extracellular Proteins as Nutrients Is Suppressed by mTORC1. Cell 2015;162:259-70.

10. Ryan MB, Der CJ, Wang-Gillam A, et al. Targeting RAS-mutant cancers: is ERK the key? Trends Cancer 2015;1:183-98.

11. Bryant KL, Stalnecker CA, Zeitouni D, et al. Combination of ERK and autophagy inhibition as a treatment approach for pancreatic cancer. Nat Med 2019;25:628-40. 